\title{
Analysis of Intra-Abdominal Pressure in Obstructive and Perforative Lesions of Gastro- Intestinal Tract
}

\author{
Kidwai ${ }^{1}$, Baral $\mathrm{RK}^{2}$, Sharma $\mathrm{A}^{3}$
}

\begin{abstract}
INTRODUCTION: Abdominal Compartment syndrome is an emerging problem in surgical patients with significant mortality reaching up to $100 \%$ in untreated patients. Intra abdominal hypertension (IAH) and abdominal compartment syndrome is common finding in traumatic and critically ill surgical patients. There are sporadic case reports in literature of intra abdominal hypertension \& abdominal compartment syndrome in general surgical patients, particularly in obstructive and perforative diseases of gastrointestinal tract (GIT). This study was done to know the pattern of intra abdominal pressure (IAP) and effect of intra abdominal hypertension (IAH) in obstructive and perforative lesions of gastrointestinal tract. Material and Method: A total number of 145 cases were included between November 2016 to October 2017. These patients were from the department of Suregery, Nepalgunj Medical College, who underwent Surgery due to the Intestinal Obstruction or perforation. RESULT: There were total 145 cases. The incidence of IAH in patients with intestinal obstruction and perforation peritonitis at presentation was $68.27 \%$. 65 (66.32\%) out of 98 patients with perforation peritonitis had IAH and 34 (72.34\%) with intestinal obstruction among 47 had IAH before surgery. There were $2.12 \%$ patients with abdominal compartment syndrome (ACS) in obstruction and 3.06\% in perforation; ACS was highest amongst traumatic perforation population accounting for $13.33 \%$ of traumatic cases. There was statistically significant derangement $(p$-value $<0.05)$ of organ function with raised IAP which showed marked improvement following surgery. Four patients died, all these patients had ACS at the time of presentation. CONCLUSION: IAH is a significant entity in obstructive and perforative lesions of GIT. ACS can occur in obstructive and perforative lesions of GIT with significant mortality.
\end{abstract}

Keywords: Abdominal compartment syndrome, decompression, intra-abdominal hypertension, Intra-abdominal pressure

\section{INTRODUCTION}

Intra abdominal pressure (IAP) and its effect on respiration and the abdominal contents has been the subject of scientific study since $19^{\text {th }}$ century. Emerson ${ }^{1}$ hypothesized reciprocal relationship between intra-thoracic pressure and IAP. Effect of raised IAP on organ function was of interest to early inventors. Bradley and Bradley ${ }^{2}$ inferred decreased renal flow and plasma filtration rate seen with increased IAP. Emerson ${ }^{1}$ demonstrated respiratory failure and death occurring with IAP above $27-46 \mathrm{~mm}$ of $\mathrm{Hg}$ in anaesthetized cats and guinea pigs. Baggot $^{3}$ recommended that tight abdominal closures and dressings be abandoned in favor of loose dressing placed on open abdomen, primarily to prevent entry of microbes in the year 1951. Many studies had showed direct relationship between raised IAP and several systemic symptoms (renal, cardiovascular, central nervous, Pulmonary etc.) which improved with abdominal decompression ${ }^{4-5}$.

Despite these early contributions, clinical and patho-physiological significance of elevated IAP went largely unrecognized for quite a long time. Intra abdominal pressure (IAP) is the pressure concealed within the abdominal cavity. Normal IAP is $0-5 \mathrm{~mm}$ of $\mathrm{Hg}$. However, in critically ill patients normal IAP is taken $5-7 \mathrm{~mm}$ of $\mathrm{Hg}$. Intra abdominal hypertension is defined by a sustained or repeated pathologic elevation of IAP $\geq 12 \mathrm{~mm}$ of $\mathrm{Hg}^{6}$.

\footnotetext{
1. Dr. Roman Kidwai

2. Dr. Ravi Kumar Baral

3. Dr. Anup Sharma
}

Address for correspondence:

Dr. Roman Kidwai

Department of Surgery

Nepalgunj Medical College \& Teaching Hospital

Nepalgunj, Banke

Email: romankidwai1@gmail.com
Abdominal compartment syndrome (ACS) is defined as sustained $\mid A P>20 \mathrm{~mm}$ of $\mathrm{Hg}$ (with or without an Abdominal perfusion pressure $(\mathrm{APP})<60 \mathrm{~mm}$ of $\mathrm{Hg}$ ) that is associated with new organ dysfunction or failure. Primary ACS is a condition associated with injury or disease in the abdomino-pelvic region that frequently requires early surgical or interventional radiological intervention. Several methods (direct, urinary bladder, rectal, gastric, inferior venacava (IVC) etc.) were implicated for the measurement of intra abdominal pressure in initial days. Intra vesicle pressure measurement, either with transducer or without it, has now become the gold standard because of its minimal invasiveness and ease in use ${ }^{7}$.

This study was conducted to identify the pattern of intra abdominal pressure in obstructive and perforative lesions of GIT and to study the effect of raised IAP in different organ or system.

\section{MATERIALS AND METHODS}

This was a prospective observational study conducted in Department of General Surgery, Nepalgunj medical college, Kohalpur teaching hospital, from November 2016 to October 2017. All patients diagnosed as a case of intestinal obstruction and perforation of GIT and undergone surgery were included. Patients who do not want to take part in the study, associated history of chronic diseases (Diabetes mellitus, significant cardiac, renal and pulmonary diseases), patients with lower urinary tract pathology where Foley catheterization was not possible, and pregnant patients were excluded. The abdominal pressure was indirectly determined by measuring urinary bladder pressure with a Foley's catheter. Patient was catheterized with a 16guage Foley's catheter. The bladder was drained and then filled with $0.5 \mathrm{ml} / \mathrm{kg}(25 \mathrm{ml})$ of sterile saline through the Foley's catheter. The catheter was connected to a saline manometer. The symphysis pubis was the zero reference, and pressure was measured in centimeters of water at end-expiration.

A conversion factor of 1.36 was used to convert the pressure into millimeter of mercury $(\mathrm{mm}$ of $\mathrm{Hg})$. Readings were taken 
preoperatively and then postoperatively at $0,6,12$, and 24 hours. The following parameters were noted blood pressure, pulse, respiratory rate, oxygen saturation, temperature, urine output, IAP, duration of surgery, preoperative findings, complications (burst abdomen, anastomotic leak, SSI) and mortality were recorded. Blood urea and serum creatinine were also noted. Intra abdominal hypertension was graded in following manner:

\section{Grading of intra-abdominal hypertension}
1. Grade I: $12-15 \mathrm{~mm} \mathrm{Hg}$;
2. Grade II: $16-20 \mathrm{~mm} \mathrm{Hg}$;
3. Grade III: $21-25 \mathrm{~mm} \mathrm{Hg}$; and
4. Grade IV: $>25 \mathrm{~mm} \mathrm{Hg}$.

The term "abdominal compartment syndrome" was used when IAH was associated with at least one newly developed organ system dysfunction or isolated pressure measurement of $>35 \mathrm{~mm}$ of $\mathrm{Hg}$. Organ system derangements were made out on the basis of following parameters:

\section{Cardiovascular system}

1. Blood pressure $<90 \mathrm{~mm} \mathrm{Hg}$ systolic or
2. heart rate $>100 /$ minute or
3. Both of the above.

\section{Respiratory system}
1. Respiratory rate $>20 /$ minute or
2. $\mathrm{SpO}_{2}<90 \%$ or
3. Patient requiring ventilator support or
4. Any two or all of the above.

Renal

$$
\begin{array}{ll}
\text { 1. } & \text { Blood urea }>40 \mathrm{mg} \% \text { or } \\
\text { 2. } & \text { serum creatinine }>1.2 \mathrm{mg} \% \text { or } \\
\text { 3. } & \text { urine output }<25 \mathrm{ml} / \text { hour or } \\
\text { 4. } & \text { Any two or all of the above. }
\end{array}
$$

\section{Statistical analysis}

The data were analyzed using Statistical Package for Social Sciences (SPSS) for Windows Version 20.

\section{RESULT}

A total of 145 patients were included in this study with the diagnosis of intestinal obstruction and perforation. Of these, 113 (77.93\%) were male and 32 (22.06\%) were female. The mean age was $37.78 \pm 18.04$ (range 2-79) years with the most common age of presentation being 45 years. 47 (32.41\%) patients had intestinal obstruction and 98 $(67.58 \%$ ) had intestinal perforations due to various causes (Figure 1).

$$
\square \text { obstruction } \square \text { perforation }
$$

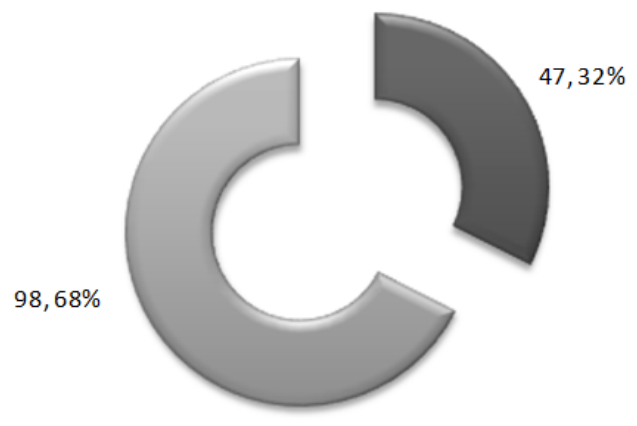

Figure 1: Incidence of Intestinal obstruction and perforation peritonitis
$83(84.69 \%)$ patients in this series were of spontaneous perforation; of these duodenal ulcers perforation (37.93\%) was the commonest finding. 15 (15.30\%) had traumatic perforations. IAH significantly varied between different diagnosis, accounting highest amongst traumatic perforation group followed by nontraumatic perforation group and small bowel obstruction. IAH at the time of admission with different causes of raised IAP is shown in Table I.

\begin{tabular}{|l|c|c|c|}
\hline \multirow{2}{*}{\multicolumn{1}{|c|}{ Diagnosis }} & \multicolumn{2}{c|}{ IAH at } & \multirow{2}{*}{ Total } \\
\cline { 2 - 3 } & Presentation & Absent & \\
\hline Appendicular perforation & 17 & 2 & 19 \\
\hline Duodenal perforation & 39 & 16 & 55 \\
\hline Gastric perforation & 0 & 1 & 1 \\
\hline Gallbladder perforation & 0 & 1 & 1 \\
\hline Ileal perforation & 6 & 1 & 7 \\
\hline Traumatic jejunal perforation & 1 & 12 & 13 \\
\hline $\begin{array}{l}\text { Traumatic jejunal \& ileal } \\
\text { perforation }\end{array}$ & 1 & 0 & 1 \\
\hline $\begin{array}{l}\text { Traumatic jejunal \& colonic } \\
\text { perforation }\end{array}$ & 1 & 0 & 1 \\
\hline Small lbowel obstruction & 33 & 10 & 43 \\
\hline Large bowel obstruction & 1 & 3 & 4 \\
\hline \multicolumn{1}{|c|}{ Total } & 99 & 46 & 145 \\
\hline
\end{tabular}

Table I: IAH at presentation

There were 10 (23.25\%) patients with small bowel obstruction and 3 (75\%) patients with large bowel obstruction who had IAH at the time of presentation. In small bowel obstruction there was a case of ACS i.e. IAH grade IV (Figure 2).

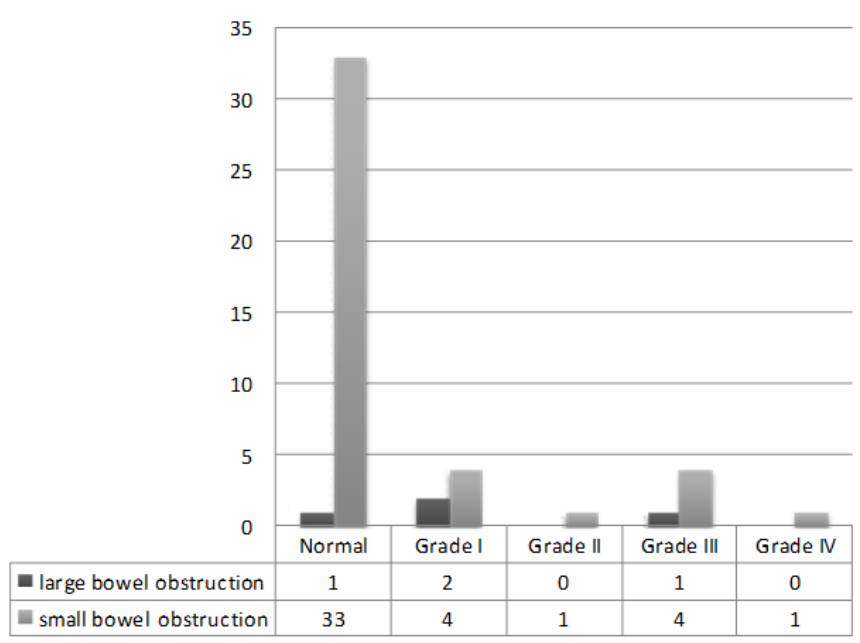

Figure 2: Preoperative grading of IAP in obstructive group $(n=47)$ 
At the time of admission there were 7 (46.46\%) patients in traumatic perforation group and $23(27.71 \%)$ in nontraumatic perforation with IAH respectively. There were 2 patients with ACS in traumatic perforation group and 1 patient with ACS in non traumatic group (Figure 3).

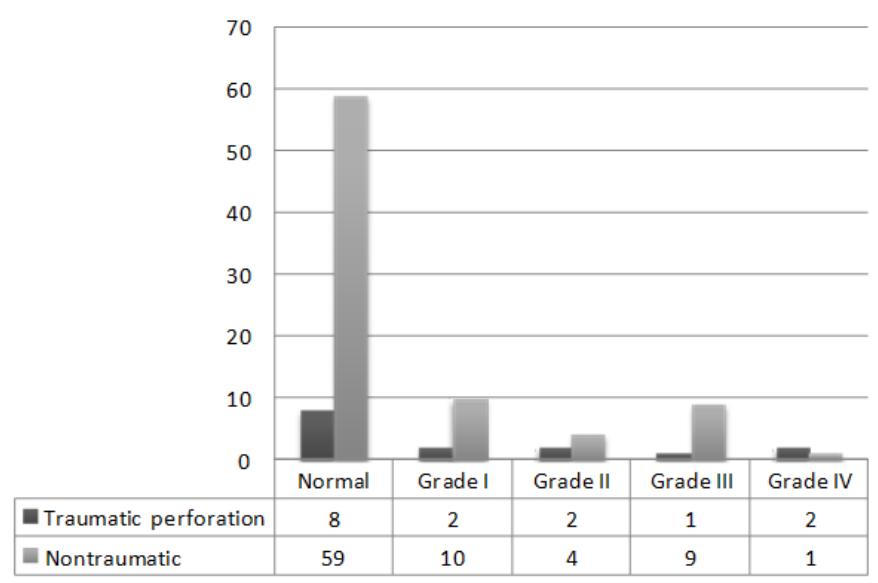

Figure 3: Preoperative grading of IAP in perforative grouph

Most of the patients showed decrease in grades of IAH after surgery. A raise in IAH was seen on $6^{\text {th }}$ postoperative hour, which again decreased in subsequent measurements at 12 and 24 hours (Table II)

\begin{tabular}{|c|c|c|c|c|c|}
\hline \multirow{2}{*}{ Category } & \multirow{2}{*}{$\begin{array}{c}\text { IAP AT admission } \\
\text { (n) }\end{array}$} & \multicolumn{5}{|c|}{ Post operative period } \\
\cline { 3 - 6 } & & IAP 0 hours (n) & IAP 6hours (n) & IAP 12 hours (n) & IAP 24hrs (n) \\
\hline Normal & $101(69.70 \%)$ & $130(89.7 \%)$ & $113(77.9 \%)$ & $125(86.2 \%)$ & $136(93.8 \%)$ \\
\hline Grade I & $18(12.4 \%)$ & $9(6.2 \%)$ & $10(6.9 \%)$ & $5(3.4 \%)$ & $1(0.7 \%)$ \\
\hline Grade II & $7(4.8 \%)$ & $3(2.1 \%)$ & $14(9.7 \%)$ & $11(7.6 \%)$ & $5(3.4 \%)$ \\
\hline Grade III & $15(10.3 \%)$ & $2(1.4 \%)$ & $7(4.8 \%)$ & $3(2.1 \%)$ & $1(0.7 \%)$ \\
\hline Grade IV & $4(2.8 \%)$ & $0(0 \%)$ & $0(0 \%)$ & $0(0 \%)$ & $0(0 \%)$ \\
\hline
\end{tabular}

Table II: IAP grading at admission and during postoperative period

Marked organ dysfunction was observed in patients with IAH. CVS and renal system were the most affected ones. CVS dysfunction was observed in 59 (40.7\%) patients at admission which after surgery decreased to 17 (11.7\%). Renal dysfunction 57 (39.3\%) was similarly profound in IAH cases at admission. Renal function also improved after surgery and patients with renal dysfunction decreased down to 16 (11\%). There was low incidence of respiratory dysfunction in pre operative period accounting for $19(13.1 \%)$ which also showed some improvement after surgery.

Four (2.75\%) patients died. There were four cases with ACS, 2(50\%) were of traumatic perforation, $1(25 \%)$ of duodenal perforation and $1(25 \%)$ of small bowel obstruction. Three patients had ACS in preoperative period and one had persistent ACS postoperatively also. All patients with ACS in our series died.

\section{DISCUSSION}

The importance of IAH and ACS is known for decades now but most of studies reported in literature have been done either in trauma cases or patients admitted in ICU. There are isolated reports of role of IAH and
ACS in surgical patients. The surgical patients at risk include those who require abdominal and retroperitoneal surgery and also when vigorous fluid resuscitation is required for shock or for any other reason. This study include group of patient who had perforative or obstructive lesions of GIT. There were $77.93 \%$ (113) male and $22.06 \%$ (32) female patients. Male dominance was seen in this study which is comparable to study conducted by Khan S. et al. ${ }^{7}$ who reported $76 \%$ (149) males and $24 \%$ (48) females. A similar male female ratio was seen in the studies conducted by Hong et al. ${ }^{8}$ (72\% males) and Meldrum et al. ${ }^{9}$ The mean \pm SD age in our study were $37.78 \pm 18.04$ years (range 2-79 year) with median age being 45 years. Most of the studies report the mean age to be higher than what we observed. Cheatham et al. have reported a mean age of $51 \pm 19$ years, Meldrum et al $39 \pm 9$ years, and Hong et al 42 years ${ }^{10}$.

Out of 145 patients in this series there were $10.34 \%$ (15) trauma patients. This is in contrast to the study by Cheatham et al who had $68 \%$ trauma patients in their study group ${ }^{10}$. This can be explained by the population selected for the study. The population pattern however matched to some extent with the group of patients reported by Khan S. et al. ${ }^{7}$ who also had included a maximum number of general surgical patients.

In patients with $\mathrm{IAH}$ at admission the mean pressure before and after decompression in our series was $19.6 \pm 5.8 \mathrm{~mm} \mathrm{Hg}$ and $7.7 \pm 1.7 \mathrm{~mm} \mathrm{Hg}$, respectively. The mean (SD) IAP in the cases studied by Surgery et al. ${ }^{8}$ before and after decompressions was $16.6 \pm 9.4 \mathrm{~mm} \mathrm{Hg}$ and $10.3 \pm 3.1$ $\mathrm{mm} \mathrm{Hg}$, respectively. Meldrum et al. reported higher values of IAP pre- and post-operatively: $27 \pm 2.3$ and $14 \pm 4.6 \mathrm{~mm} \mathrm{Hg}$, respectively ${ }^{9}$. This can be explained by the observation that in our study, $88.32 \%$ of the patients had perforation and obstruction leading to elevated IAP which, after decompression and removal of fluid and gas, returned to normal level immediately.

The incidence of IAH in our study was $32 \%$ at admission and $20 \%$ at 6 hours post-operatively. The incidence of ACS was 3.06\% (3 out of 98 patients) in perforative lesions, $2.12 \%$ (1 out of 47 patients) in obstructive lesions and $13.33 \%$ ( 2 out of 15 patients) in trauma patients. Three out of four patients with ACS were in pre operative period and one was in post operative period. The incidence of IAH and ACS reported by various authors before and after decompression ranged from 2 to $78 \%$ and 0.5 to $36 \%$, respectively and depends on the study population and the values used to define these entities ${ }^{10}$. The lower incidence observed in this series was because this study included low risk as well as high-risk patients, whereas most of the previous studies confined data collection to high-risk patients.

While the latter approach ensures a good yield of patients with ACS, it may result in a very high incidence compared with that seen clinically in overall general population. Furthermore, such an approach potentially misses those patients who are not at high risk and yet may have Multiple Organ Dysfunction Syndrome (MODS) falsely attributed to sepsis or irreversible shock when in fact they have unrecognized ACS. By measuring the IAP prospectively in all patients, this study obtained true overall incidence. Also, an incidence of $2.75 \%$ in overall study population is significant enough to warrant further investigation in this group.

No significant association was found between IAP at any point of time and occurrence of leakage $(P>0.05)$. IAH at admission and persistence raise of IAP in post operative period particularly at 6 hours and later had detrimental effect both on morbidity and mortality. Cheatham et 
al had found that elevated IAP alone does not have sufficient sensitivity or specificity to be used as a predictor of mortality ${ }^{10}$. But in our patients, elevated IAP pre-operatively and post-operatively at 6 hours was found to independently predict the occurrence of death ( $P$ $<0.05$ ) but not at post operatively at 0,12 and 24 hours $(P>0.05)$.

The mortality rate in ACS group was $100 \%$ despite decompression. In a retrospective study conducted by Britt et al. in 2005 reported overall mortality of $60 \%$ and $43 \%$ after decompression. ${ }^{11}$ The high mortality in our study despite decompression could due to early fulminant multiple organ dysfunction syndrome (MODS) or delay in decompression as the IAP readings were taken at 0, 6, 12 and 24 hours post-operatively with no reading in between. Hence, a more frequent IAP monitoring is recommended, at least in high-risk patients.

\section{CONCLUSION}

Patients with intestinal obstruction and perforation had elevated IAP at presentation. IAP is a significant predictor of mortality and morbidity, in patients with obstructive and perforative lesions of GIT.IAH has a detrimental effect on various organ functions. Decompression leads to improvement in all the parameters. ACS was associated with very high mortality rate in this study. A more frequent monitoring of IAP with prompt decompression may be helpful in decreasing the morbidity and mortality rate. Further studies are required to establish a screening protocol in patients with obstructive and perforative lesions of GIT to detect and manage cases of IAH and ACS.

\section{REFERENCES}

1. Emerson $\mathrm{H}$ : intra abdominal pressures. Arch internal med 1911; 7:745-84.

2. Bradley SE, Bradley GP: The effect of increased abdominal pressure in renal function. J Clin Invest 1947; 26:1010-5.

3. Baggot MG: Abdominal blow out a concept. Curr. Res Anaesthesia Analg 1951; 30:295-9.

4. Korn IL, Hartman PK, Nolan SP: the measurement of intra abdominal pressure as a criterion for abdominal reexploration. Ann Surg 1984; 199:28-30.

5. Danieal De Backer:Abdominal compartment syndrome. Crit Care 1999, 3(6): R103-4.

6. World Society for Abdominal Compartment Syndrome Consensus: Definitions and Recommendations [http://www.wsacs.org].

7. Khan S, Verma AK, Ahmad SM and Ahmad R. Analyzing intra abdominal pressures and outcomes in patients undergioing emergency laparotomy. J emmerg trauma shock. 2010; 3(4):31825.

8. Sugrue M, Buist MD, Hourihan F, Deane S, Bauman A, Hillman K. Prospective study of intra-abdominal hypertension and renal function after laparotomy. Br J Surg. 1995;82:235-8.

9. Meldrum DR, Moore FA, Moore EE, Franciose RJ, Sauaia A, Burch JM. Prospective characterization and selective management of the abdominal compartment syndrome. Am J Surg. 1997;174:667-73.

10. Cheatham ML, White MW, Sagraves SG, Johnson JL, Block EF. Abdominal perfusion pressure: a superior parameter in the assessment of intraabdominal hypertension. J Trauma. 2000;49:621-7.

11. Britt RC, Gannon T, Collins JN, Cole FJ, Weireter LJ, Britt LD. Secondary abdominal compartment syndrome: risk factors and outcomes. Am Surg. 2005;71:982-5. 